\title{
Angle-dependent time-shift modeling by 3D ray-tracing
}

\author{
Bruno Pereira Dias*, Vitor Leal de Mello, Filipe Borges, and Roberto de Melo Dias (PETROBRAS)
}

Copyright 2021, SBGf - Sociedade Brasileira de Geofísica

This paper was prepared for presentation during the $17^{\text {th }}$ International Congress of the Brazilian Geophysical Society held in Rio de Janeiro, Brazil, 16-19 August 2021.

Contents of this paper were reviewed by the Technical Committee of the $17^{\text {th }}$ International Congress of the Brazilian Geophysical Society and do not necessarily represent any position of the SBGf, its officers or members. Electronic reproduction or storage of any part of this paper for commercial purposes without the written consent of the Brazilian Geophysical Society is prohibited.

\begin{abstract}
We developed an algorithm to calculate angle-dependent time-shifts with a $3 \mathrm{D}$ ray-tracing modeling engine. Two subsurface 4D models helped us evaluating the benefits of the methodology for scenarios of fluid and pressure changes, as well as geomechanical effects in the overburden. The developed algorithm is computationally efficient, and the results show good agreement with crosscorrelation time-shifts from 3D full-wave modeled data. The proposed methodology addresses a major simplification in time-shift interpretation and has the potential to improve 4D studies, in comparison with the standard vertical propagation approach. The mitigation of uncertainties, avoiding interpretation pitfalls, as well as the use of anglestack time-shifts, add up to a more robust interpretation of time-lapse data.
\end{abstract}

\section{Introduction}

A time-lapse modeling study usually comprises the simulation of reservoir production scenarios (including fluid saturation, pressure changes and other effects), from which it is possible to estimate measurable seismic attributes after a petroelastic modeling step (PEM). Some seismic measurements (e.g. 4D time-shifts) commonly encompass the combined effects of velocity and displacements changes (Landrø and Stammeijer, 2004).

The current industry standard for time-shift modeling is quite simple: it is often considered that the seismic wavefield is propagated vertically and registered in a zero-offset scheme, despite the tridimensional character of seismic experiments with the customary large offsets between sources and receivers.

The goals of this study are twofold. First, we introduce our proposed algorithm to model the expected 4D time-shift in an experiment with non-vertical seismic propagation. After that, we present synthetic examples to show the impact of the vertical versus non-vertical hypothesis in time-shift estimation. The examples demonstrate the benefits of the 3D modeling approach in terms of accuracy and fidelity. The outcome of this algorithm can subsidize the analysis of pre-stack or partial stack time-shift volumes. Such data becomes an additional source of information for the process of seismic interpretation, reservoir monitoring and characterization, and geomechanical studies, providing important information for reservoir management.

\section{Method}

The time-shift modeling consists in evaluating the difference of the two-way time propagation $(\Delta T)$ between a monitor and a base survey along ray-paths $l_{m}$ e $l_{b}$ (Kudarova et al. 2016):

$$
\Delta T=\oint_{l_{m}} \frac{d l}{v_{m}}-\oint_{l_{b}} \frac{d l}{v_{b}}
$$

where $v_{b}$ and $v_{m}$ stand for base and monitor velocity, $l_{b} \mathrm{e}$ $l_{m}$ are the ray path for the base and monitor surveys, and the symbol $\oint d l$ means the integration operator along the ray path. Landro and Stammeijer (2004), Roste et. al. (2006), and Kudarova et al. (2016) report the use of straight ray-paths to evaluate Eq. (1). However, this approximation in unsatisfactory in at least two relevant aspects (see Figure 1):

1. This hypothesis is only valid for thin-layered property changes, with only one reservoir in the section and without geomecanical effects on the overburden.

2. For the correct modeling, it is necessary to determine which rays are recorded by the seismic acquisition apparatus, contributing for the image formation. Raytracing provides accurate subsurface angles estimation.

To overcome these issues, we implemented a 3D solution based on ray-tracing to model time-shifts by angle (Figure 2). A fast and parallelized engine executed in a coarse-grid velocity model addresses the elevated computational cost of shooting rays for every voxel in the model. On the other hand, to maintain the high precision requirement of timeshift calculations, we execute the cell-by-cell integration, via a fast line algorithm (Siddon, 1985), in densely sampled base and monitor velocity models.
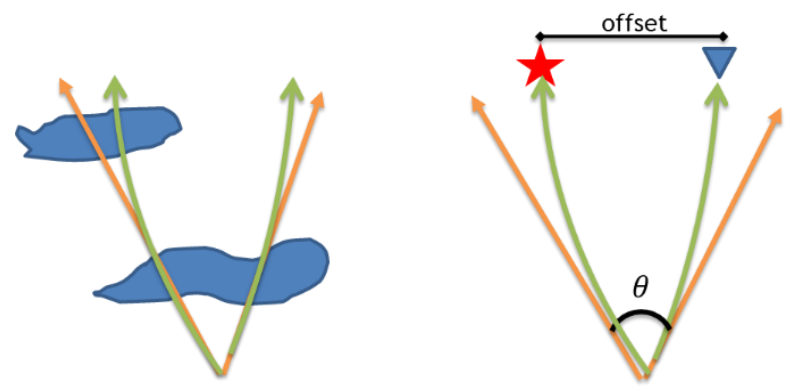

Figure 1: Cases where the straight ray path approach (orange lines) is unsatisfactory. Left, stacked reservoirs in the same section and right, to determine the ray paths recorded by the seismic acquisition apparatus. 


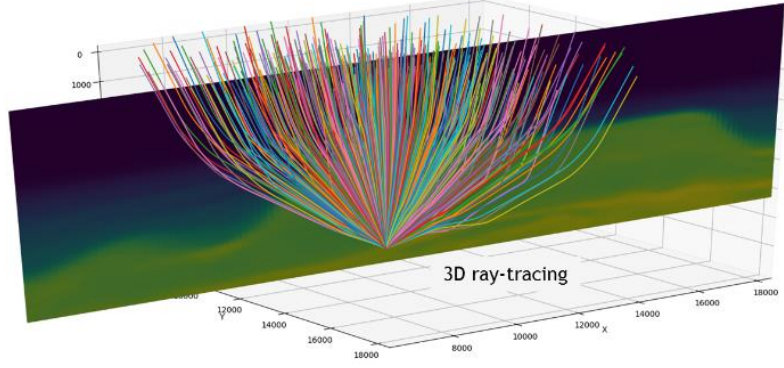

Figure 2: Example of the 3D ray traces used for the timeshift modeling.

\section{Toy-Examples}

To illustrate the impact of time-shift by angle, we start by applying our algorithm in three simple scenarios (Figure 3):

1. Depletion: consists of a velocity increase of $150 \mathrm{~m} / \mathrm{s}$ in a reservoir of $1500 \mathrm{~m}$ length and $100 \mathrm{~m}$ thick.

2. Gas injection: consists of a velocity decrease of 300 $\mathrm{m} / \mathrm{s}$ in a region of $100 \mathrm{~m}$ length and $50 \mathrm{~m}$ thick.

3. Combined effects: the superposition of the two previous models.

\section{Depletion}

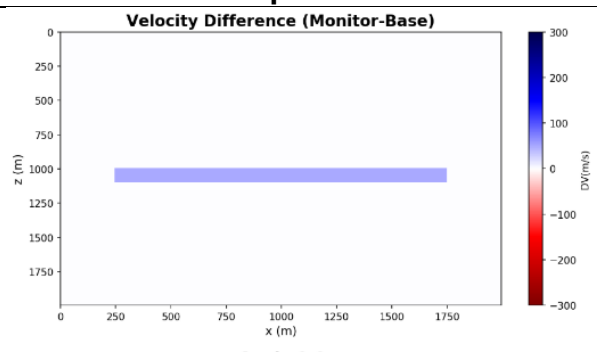

Angle 0-0 0

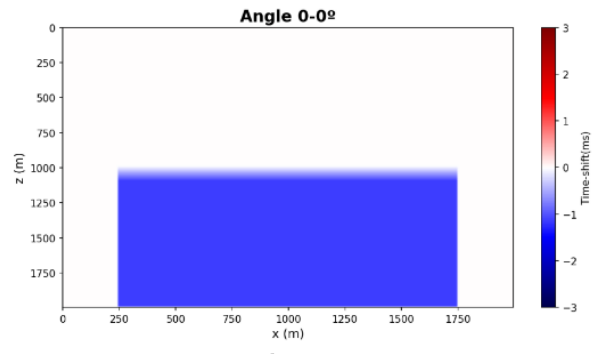

Angle 0-159
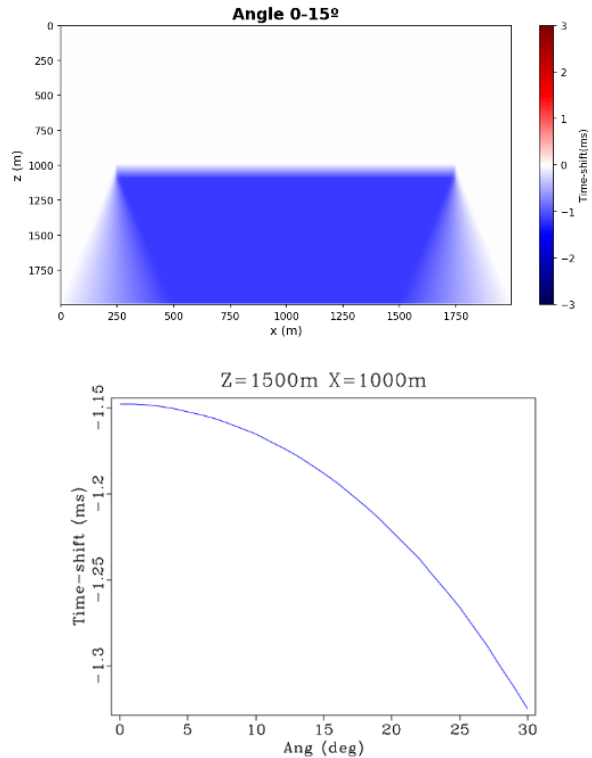

Gas injection
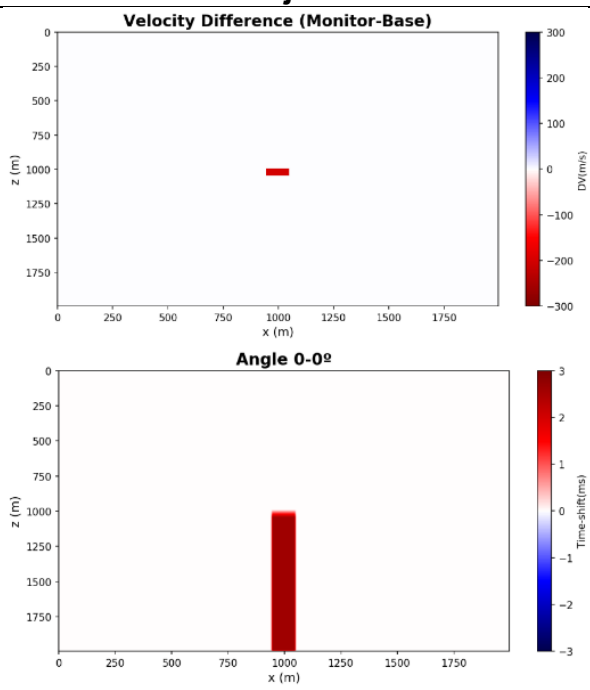

Angle 0-15o
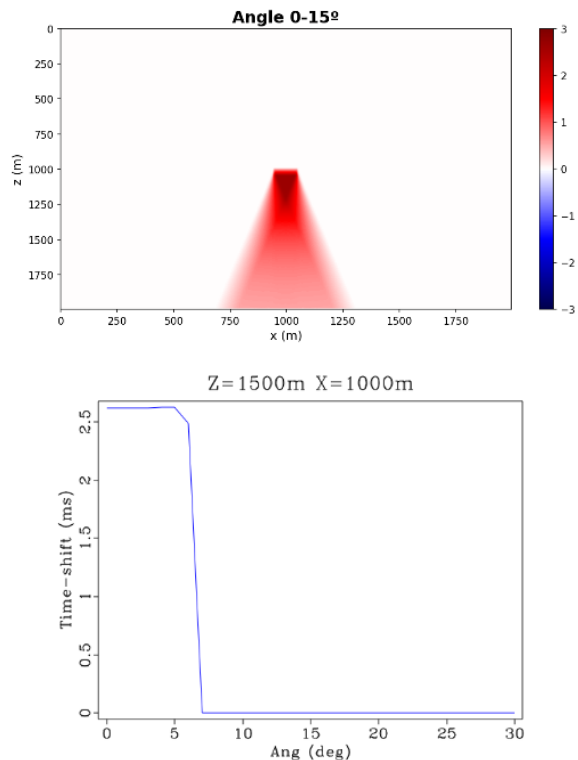

Combined effects
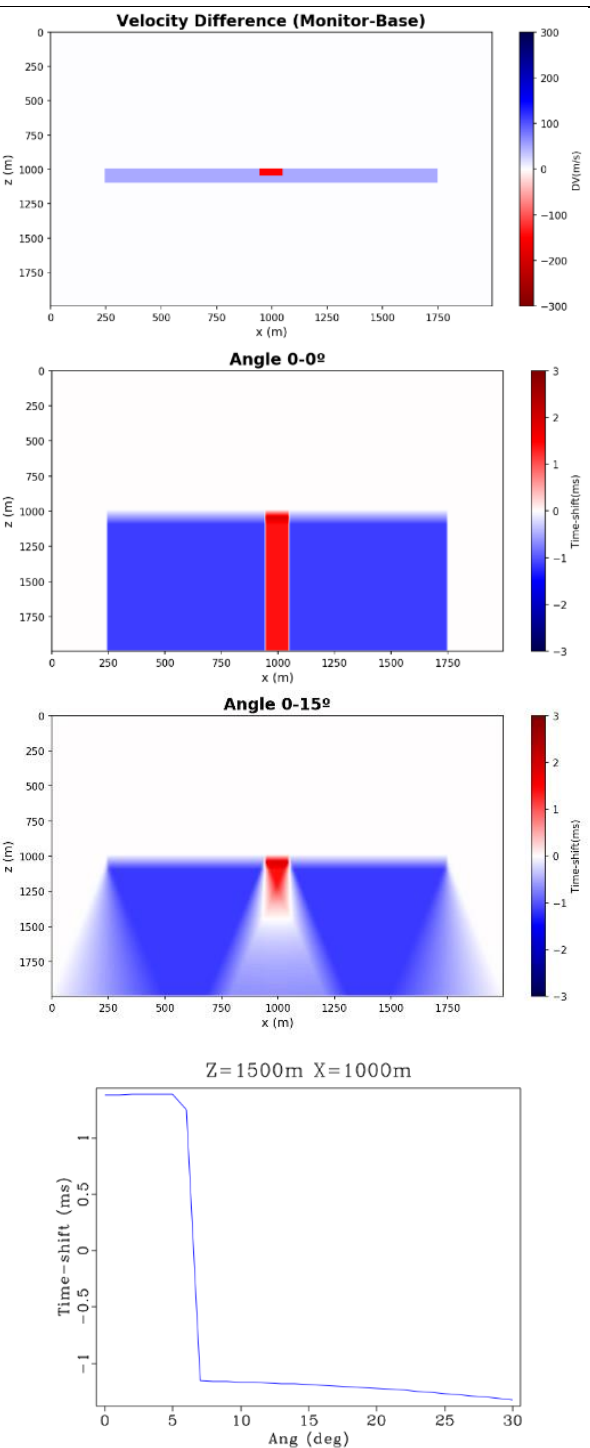

Figure 3: Toy-examples for time-shift calculation on a pressure depletion model (left), a gas injection model (center), and combined effects (right). Rows from top to bottom are velocity difference (monitor-base), time-shift by vertical propagation, time-shift by angle (0-15\%), and time-shift by angle at $z=1500 \mathrm{~m}, \mathrm{x}=1000 \mathrm{~m}$ (center of the model). 
For a long 4D velocity anomaly (depletion example), the magnitude of the angle-dependent time-shift measured below the anomaly (and far from its borders) is increased by a factor of $\cos ^{-1}(\theta)$ :

$$
\Delta T(\theta)=\frac{\Delta T_{0}}{\cos (\theta)} .
$$

The increase happens because a tilted ray-path crosses a larger section of changed velocity, and it is illustrated in Figure 3, where the changes in velocity and the angledependent time-shifts for the three scenarios are shown. The color convention used here follows the standards proposed by Stammeijer and Hatchell (2014), where cold colors are associated to hardening (negative time-shifts) and warmer colors, to softening (positive time-shifts).

On the other hand, a dimming effect, i. e. reduction of the magnitude of the time-shifts, is observed at the borders of the reservoir, or in localized anomalies, as in the gas injection example (Figure 3, center column). The explanation in simple: only part of the ray path (source- or receiver-side) crosses the $4 \mathrm{D}$ velocity anomaly, and hence the total contribution to the time-shift is smaller.

The combination of both effects reveals the complex behavior of the time-shift as function of angle (Figure 3, rightmost column), which analytic vertical models fail to account for.

\section{Application 1: Saturation and pressure changes}

The first application focus on saturation and pressure changes in the reservoir. The 4D properties used as input for time-shifts modeling result from a petroelastic modeling workflow based on reservoir flow simulation of a Santos Basin pre-salt field, calibrated with well-logs and lab measurements (Mello, et al., 2019). The model outside the reservoir is derived from full waveform inversion and full band acoustic impedance, subsequently mapped to $V_{p}, V_{s}$, and density via chained formulas. The 4D anomalies present in the model include complex fluid fronts like alternated water and gas streaks and reinjected gas (Deplante et al 2019).

Using the proposed methodology, we computed time-shift volumes for partial-stacks with angles ranges of $0-12^{\circ}, 13-$ $25^{\circ}, 26-38^{\circ}$, and $39-51^{\circ}$. For this work, the time-shifts for each angle sub-stack consist of a simple average of angleby-angle values. In the future, we pretend to weight each angle sub-stack by the hit count and asymptotic seismic amplitude.

To validate the results, we compare the modeled outcome with time-shifts estimated by a cross-correlation algorithm. The input seismic data are pre-stack reverse-time migration (RTM) volumes, obtained from full-wavefield elastic modeling on base and monitor scenarios. One would expect that the time-shifts calculated by 3D ray tracing are more accurate than the simple vertical assumption. Nevertheless, when comparing the results, it is important to be aware that there are limitations related to the cross-correlation algorithm used to measure time-shift - the presence of numerical 3D and 4D noise coming from the full-elastic modeling, for example.

Figure 4 shows depth sections and depth-slices for modeled and estimated time-shifts. The angle-dependent effects are clearly visible when comparing near/mid vs. far/ultra-far angles. Some dimming can be noted below the position of the gas-injecting wells (strong, red-colored anomalies), specially for the farther angles. The increase of time-shift magnitude vs. angle due to reservoir depletion is measurable, although visually its effect is subtle. The simplified vertical propagation assumption result is close to the near angle results $\left(0-12^{\circ}\right)$, which means that restricting modeling to this approximation can lead to incomplete and even misleading - interpretation of time-shift anomalies. 

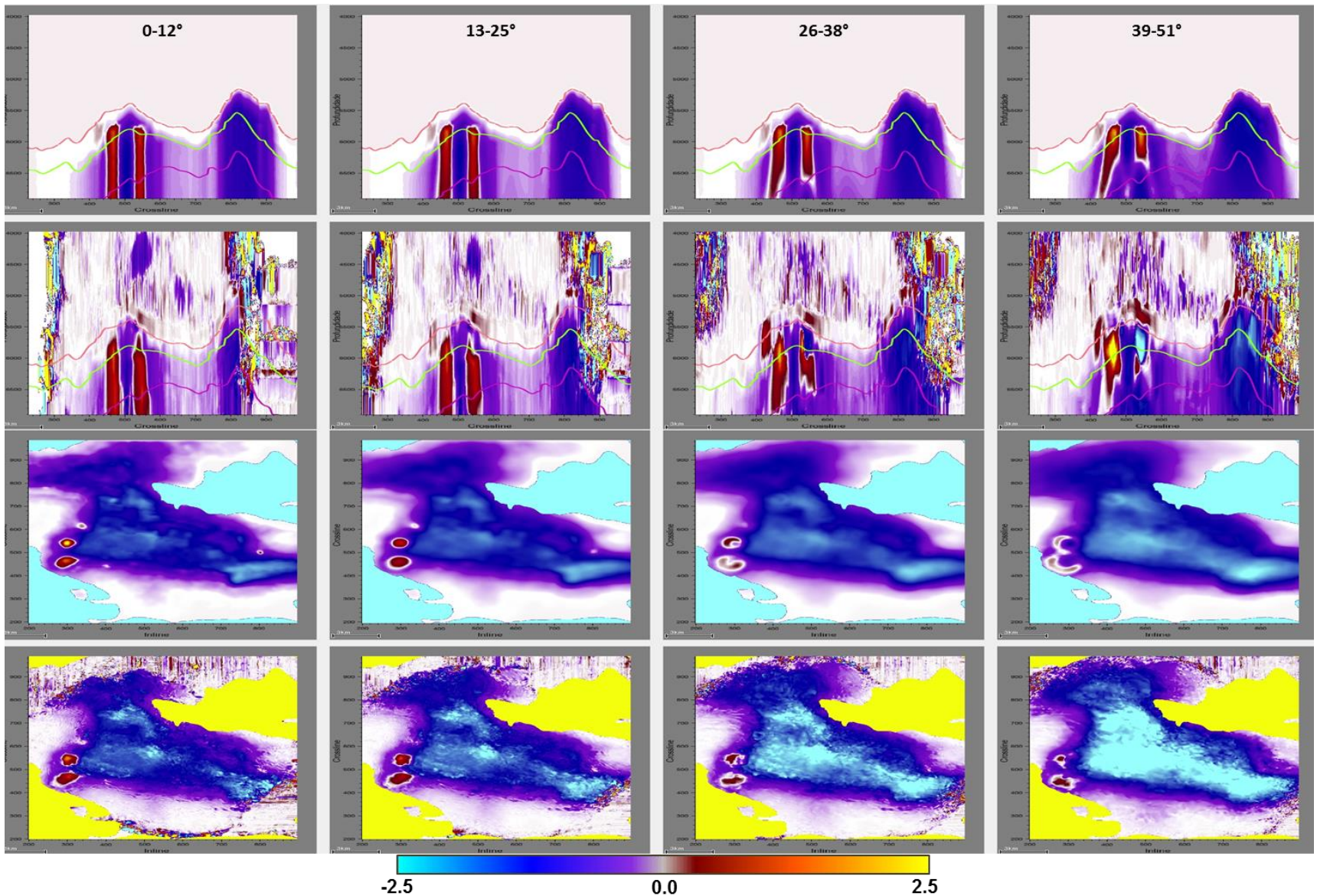

Figure 4: Time-shift modeling for a reservoir production scenario. Rows from top to bottom are: depth section of the modeled time-shift, estimated time-shift by cross-correlation, depth-slice of the modeled time-shift, estimated time-shift by cross-

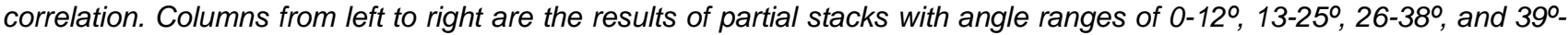
$51 \stackrel{0}{ }$

\section{Application 2: Geomechanical effects in the overburden}

In the second application example, we investigate the benefits of the proposed methodology in a scenario where reservoir production induces geomechanical effects, such as rock strain and, consequently, velocity changes at the reservoir and surrounding rocks. Although the velocity variations are smaller than the previous case, the extension of the perturbations is far greater. Consequently, the ray trajectories play a more important role in the determination of the computed time-shifts.

For this application, two different scenarios of geomechanical responses to reservoir production are considered. Scenario 1 and 2 differ on the of Young and Poisson values in the evaporitic section, corresponding to "soft" and "hard" properties, respectively. The modeled time-shifts clearly capture these different seismic responses. In Scenario 1, the positive and negative timeshift anomalies have greater magnitudes in comparison with Scenario 2. In addition, the compression effect is localized near the vicinities of the reservoir in the first scenario. The time-shift modeling results at the top of reservoir are shown in Figure 5. The positive values delimit the regions where dilation occurred due to reservoir depletion. The negative values are associated with the compression of the surrounding rocks (stress-arching effect) (Herwanger and Koutsabeloulis, 2011).

The comparison between vertical propagation and 3D raytracing approach is even more dramatic here than in the previous application. The 3D ray-tracing modeling shows significant differences for both scenarios. However, considering that this result is closer to the one that would be obtained in a real 4D seismic data, and if supposedly only vertical propagation time-shift modeling were provided to subsidize interpretation, the possible implications could be:

1) If Scenario 1 materialized, it would not be possible to conclude safely which scenario is true, since the 3D ray-tracing result lies in between the two vertical propagation scenarios in terms of magnitude and shape of anomalies.

2) If Scenario 2 materialized, neither scenarios would be confirmed as true. The 3D ray-tracing modeling shows time-shift magnitudes weaker than those predicted by 
the vertical propagation scenarios, resulting in vanishing or shape alteration of the main anomalies.

In the vertical section view (Figure 6), the presence of several discrepancies could put in doubt the quality of the 4D data in some portions of the volume. Among the differences, there are noticeable magnitude variations, polarity inversion and asymmetries. An evident example happens below the reservoir, where vertical propagation modeling does not predict a time-shift anomaly.

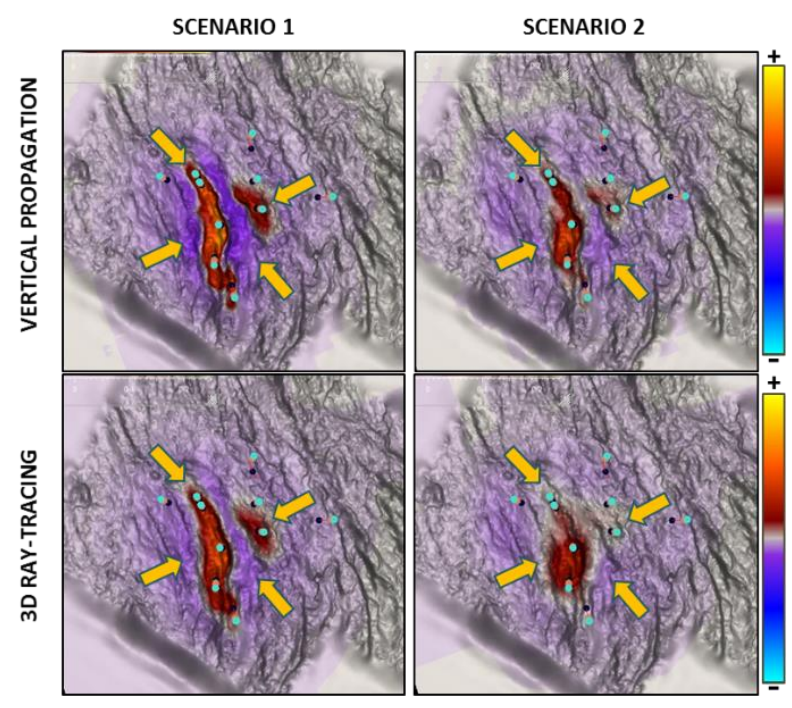

Figure 5: Time-shift horizon slices for two geomechanical simulation scenarios ("soft" and "hard" properties). There is a significant difference between the results considering vertical propagation and $3 D$ raytracing.

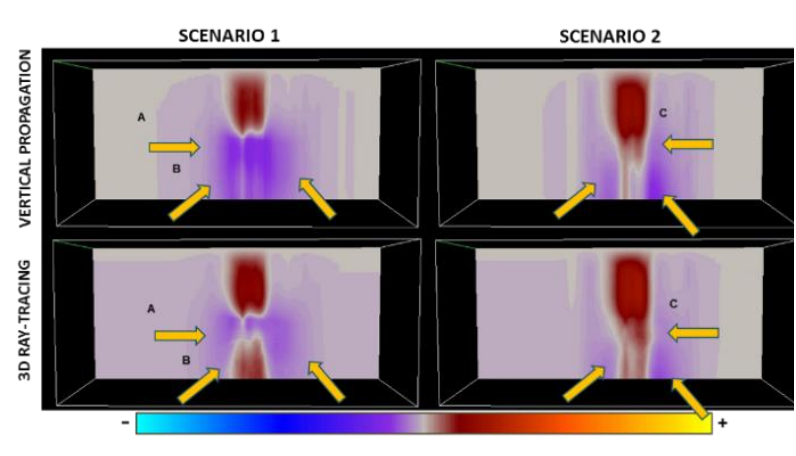

Figure 6: Time-shift depth sections for two geomechanical simulation scenarios. Noticeable discrepancies are $(A)$ magnitude variations, $(B)$ polarity inversion, and (C) asymmetries.

\section{Conclusion}

A 3D ray-tracing algorithm for time-shift modeling predicts patterns not contemplated by vertical propagation modeling (the industry standard). Dimming effects occurs at the border of the reservoirs or in laterally restricted anomalies. Time-shift magnitude increases with angle in the central area of extensive and thick anomalies. The results for the scenario of fluid/pressure changes show good correspondence with measured time-shifts. The case with geomechanical effects in the overburden puts in evidence some dramatic differences, since the significant extension of the time-shift anomalies plays an important role.

With the growing use of full azimuth, large offsets, ocean bottom nodes and even permanent or semi-permanent sea-bottom systems for seismic monitoring, a vast number of seismic volumes is available, but many are not used in the current workflows. Moving beyond zero-offset is crucial for the understanding of pre-stack, angle-stack, and azimuthal analysis of time-shifts. Taking advantage of this information might be a powerful step to maximize the business case of $4 \mathrm{D}$ projects. We expect that this more rigorous algorithm may unveil further usefulness of the available information in time-shifts for field management and HSE purposes.

\section{Acknowledgments}

The authors thank PETROBRAS for authorizing this publication.

\section{References}

Deplante, C., Costa, M., Sebastião dos Santos, M., de Melo Dias, R., Radefeld Meirelles, B., da Silva Araujo, S., Chagas Garcia., D., Sansonowki, R., Using full wave seismic modeling to test 4D repeatability for Libra pre-salt field, 2019, Sixteenth International Congress of the Brazilian Geophysical Society, SBGf.

Herwanger, J. and Koutsabeloulis, N., 2011, Seismic Geomechanics: How to Build and Calibrate Geomechanical Models using 3D and 4D Seismic Data: EAGE Education Tour Series 5.

Kudarova, A., Hatchell, P., Brain, J., MacBeth, C., 2016, Offset-dependence of production-related 4D time-shifts: real data examples and modeling: SEG International Exposition and 86th Annual Meeting.

Landrø, M., and J. Stammeijer, 2004, Quantitative estimation of compaction and velocity changes using 4D impedance and travel time changes: Geophysics, 69, 949957.

Mello, V. L. D., Santos, M. S. D., Penna, R. M., Rosseto, J. A., and Deplante, C., 2019, 4D Petroelastic modeling for a Brazilian pre-salt field: what to expect from interpretation?. 81st EAGE Conference and Exhibition

Røste, T., Stovas, A., Landrø, M., 2006, Estimation of layer thickness and velocity changes using 4D prestack seismic data: Geophysics, 71, S219-S234.

Siddon R. L., 1985, Fast calculation of the exact radiological path for a three-dimensional CT array: Medical Physics 12 (2), p. 252-255.

Stammeijer, J., and P. Hatchell, 2014, Standards in 4d feasibility and interpretation: TheLeading Edge,33, 134140. 